\title{
ACTIVIDADES DE LA DIVISIÓN DE ÁRABE DE LA UNIVERSIDAD DE ALICANTE (1988)
}

\author{
Por \\ MÍKEL DE EPALZA
}

CAMBIO DE NOMBRE DEL DEPARTAMENTO. A petición de la Junta del Departamento, la Junta de Gobierno de la Universidad de Alicante aprobó el cambio de nombre del Departamento de «Filologías Árabe, Catalana y Francesa" en Departamento de "Filología Catalana, Estudios Árabes e Islámicos y Filología Francesan, correspondiente a las denominaciones de las tres áreas de conocimiento del Departamento y de sus divisiones departamentales.

MARCELINO VILLEGAS, PROFESOR TITULAR NUMERARIO. Tras su correspondiente concurso, tomó posesión el 10 de noviembre de 1988 de la plaza de Profesor Titular Numerario de Estudios Árabes e Islámicos el Dr. Marcelino Vil!egas González, anteriormente profesor en Madrid, Bagdad y Orán, y, desde 1987, Profesor Titular interino de la Universidad de Alicante.

LA DRA. MARGARITA LA CHICA Y EL DR. ALBERTO ARAUJO, COLABORADORES HONORÍFICOS. Han sido nombrados, por el Rectorado de la Universidad de Alicante, Profesores Colaboradores Honoríficos del Departamento, los Dres. Margarita La Chica Garrido, profesora jubilada de árabe, y el Dr. Alberto Araujo Fernández, profesor de hebreo y licenciado en Filología Semítica por la Universidad de Madrid.

BECAS DE INVESTIGACIÓN. Vinculadas a la División de Estudios Árabes e Islámicos, han sido concedidas en 1988 a María Sol Cabello García (DGICYT), Luis Fernando Bernabé Pons (Generalitat Valenciana) y Francisco Franco Sánchez (UNED). 
INTERCAMBIO DE BECARIOS DE LA UNIÓN DE UNIVERSIDADES DEL MEDITERRÁNEO. La Unión de Universidades de Mediterráneo, con sede en Bari (Italia), concedió, para 1988, sendas becas a Massimo Laria, de la Universidad de Venecia, y a María Magdalena Martínez Almira, de la de Alicante, para realizar estudios arabísticos respectivamente en España y en Italia, bajo la dirección de los profesores Francesco Castro y Míkel de Epalza.

CÁTEDRA POR PROGRAMA P.R.O.P.I.O. El Programa Universitario PROPIO (Programa de Oferta de Plazas con la Investigación como Objetivo), del Ministerio de Educación y Ciencia, tras valoración de peticiones a nivel nacional, ha concedido una Cátedra de "Estudios Árabes e islámicos" a la Universidad de Alicante, en consideración a la labor de su equipo de investigación en esa área de conocimiento y particularmente a la del candidato Dr. Míkel de Epalza.

PROGRAMA DE INVESTIGACIÓN DE LA D.G.I.C.Y.T. Ha sido concedido, en fecha del 26-07-88, a la Universidad de Alicante la financiación de un Programa de investigación de la DGICYT (Dirección General de Investigación, Ciencia y Técnica), del Ministerio de Educación y Ciencia, sobre "Corpus historiográfico y crítica literaria de la literatura hispano-árabe», cuya investigadora principal es la Dra. María Jesús Rubiera Mata.

PROGRAMA «EL HOMBRE Y EL AGUA EN EL MEDITERRÁNEO Y EL MAR NEGRO". Dentro del marco de este programa internacional (ver SHARO AL-ANDALUS. ESTUDIOS ÁRABES, vol. 4, p. 375), cuyo coordinador nacional en España es el Dr. Epalza, se han realizado: en Alicante, un congreso sobre "Avenidas fluviales e inundaciones en la cuenca del Mediterráneo", por el Instituto de Geografía de la Universidad (18-21 mayo 1988), y en Zaragoza, sobre "La Ciudad Islámica» (24 junio 1988). El libro de actas del simposio internacional «Aguas y poblamiento musulmán", que se había realizado en febrero de 1987 dentro de este programa ha sido ya editado (ver Sección Bibliografía, en este volumen de la revista).

CONGRESO INTERNACIONAL «LA CIUDAD ISLÁMICA». Co-dirigido por los Dres. José Luis Corral, de la Universidad de Zaragoza, y Míkel de Epalza, de la de Alicante, y organizado por la Institución «Fernando el Católico» de la Diputación General de Aragón, se celebró en Zaragoza del 1 al 4 de diciembre un Congreso Internacional sobre "La Ciudad Islámica" con más de 30 ponencias y comunicaciones y una asistencia de más de 230 congresistas. Presentaron ponencias los Sres. Rubiera y Epalza y comunicación el Sr. Franco, de la división departamental de Estudios Árabes e Islámicos de la Universidad de Alicante (ver Noticia aparte).

PROGRAMA «ERASMUS». Dentro del programa europeo «ERASMUS» de intercambios de profesores y estudiantes, han sido aprobadas diversas actuaciones y visitas de profesores de la División de Estudios Árabes e Islámicos de la Universidad de Alicante y de los departamentos de árabe de las Uni- 
versidades de Mons (Bélgica), Montpellier y Toulouse (Francia) y Perugia, Roma y Nápoles (Italia), visitas que se realizarán en 1989.

CERTIFICADO PARA POSTGRADUADOS DE «ESTUDIS ARABICS VALENCIANS" EN LA UNIVERSIDAD DE VALENCIA. En coordinación con el Departamento de Filología Catalana de la Universidad Estudis Generals de Valencia, la División de Estudios Árabes e Islámicos de la Universidad de Alicante ha organizado para el curso 1988-1989 un curso para post-graduados «Estudis Aràbics Valencians".

CURSO DE DOCTORADO EN OVIEDO. El Dr. Epalza impartió, a principios de mayo de 1988, en el Departamento de Filología Románica de la Universidad de Oviedo, un curso de doctorado sobre "Estudios Árabes e Hispánico: Historia, religión, urbanismo, toponimia y literatura». La Dra. Rubiera dio una conferencia en dicho Departamento sobre "Una nueva teoría sobre la lengua de las jarchas».

CURSO EN LA UNIVERSITAT CATALANA D'ESTIU. EI Dr. Epalza dio, en agosto de 1988, un curso de verano en la Universitat Catalana d'Estiu en Prades (Francia), sobre "La cultura àrab i els Paísos Catalans".

JURADO DEL PREMIO NACIONAL DE TRADUCCIÓN. El Dr. Villegas fue nombrado miembro de la comisión que otorgó los Premios Nacionales de Traducción 1988, del Ministerio de Cultura.

TESIS DOCTORAL EN LA UNIVERSIDAD DE GRANADA. EI 6 de junio fue leida en la Universidad de Granada la tesis doctoral sobre la Escuela de "Lecturas Coránicas» de Denia, por Wilhelmina Wagner de Al-Gannabi. La nueva Doctora es antigua alumna de la Universidad de Alicante y su tesis fue codirigida por el Dr. Epalza.

TRIBUNALES DE TESIS DOCTORALES. La Dra. Rubiera formó parte de la Comisión que juzgó la tesis doctoral de Abu-Bakr Abdelwahhab "La poesía almohade: el madîh o panegírico", en la Universidad Complutense de Madrid. El Dr. Villegas formó parte de la Comisión que juzgó la tesis doctoral de Nieves Paralela Alonso «El viaje árabe a España en época moderna y contemporánea (desde el siglo XVII hasta 1939)" en la Universidad Autónoma de Madrid. El Dr. Epalza formó parte de la Comisión que juzgó la tesis doctoral de Pere Santonja "Arnau de Vilanova: els escrits religiosos i la Inquisició» en la Universidad de Alicante.

TESINA DE LLÚCIA MARTÍN. El 22 de octubre de 1988 leyó su Memoria de Licenciatura Llúcia Martín Pascual, colaboradora habitual de esta revista y División Departamental, sobre «El deute de la História de Jacob Xalabín amb la tradició oriental. Extensió i límits". Formaron parte de la Comisión calificadora los Dres. Rubiera, Epalza y Rafael Alemany. Obtuvo la máxima calificación.

PREMI "MENÉNDEZ Y PELAYO». El Premi "Menéndez y Pelayo» de In- 
vestigación 1988, del Institut d'Estudis Catalans, ha sido concedido a los Dres. María Jesús Rubiera y Míkel de Epalza por su libro "Los nombres árabes de Benidorm y su comarca». El Premi les fue entregado en sesión solemne del Institut, en Barcelona, el 25 de octubre.

PREMI INTERNACIONAL «RAMON LLULL»AL P. BURNS. EI Premio Internacional de Investigación «Ramón Llull» 1988 fue concedido por la Generalitat de Catalunya al P. Robert Ignatius Burns S. J., profesor de la Universidad de California - Los Ángeles, historiador especialista en el siglo XIII valenciano y asiduo colaborador de esta revista y de la División departamental de Estudios Árabes e Islámicos de la Universidad de Alicante (ver SHARO ALANDALUS. ESTUDIOS ÁRABES vol. 3, pp. 295 y 296, y artículos en volúmenes 1, 4 y 5). El Premi le fue entregado en Barcelona el 10 de mayo.

CONCURSO AL PREMIO "AL-SHAFRA». Organizado por la Asociación Cultural «Muhammad Al-Shafra», que dirige el Catedrático de Cirugía Justo Medrano, de la Universidad de Alicante, otorgó su Primer Premio a los becarios de este Departamento Francisco Franco y María Sol Cabello (ver a continuación Noticia especial).

LIBROS PUBLICADOS. Los profesores de árabe de la Universidad de Alicante, además de artículos científicos y de divulgación y de comunicaciones en congresos científicos, han publicado en 1988 los libros que se recensionan en un apartado especial de la Sección Bibliografía de este volumen de la revista SHARQ AL-ANDALUS. ESTUDIOS ÁRABES.

CO-EDITOR DE LA REVISTA «AL-MASĀQ». El Dr. Epalza ha sido nombrado "Associate Editor» de la revista de estudios árabes mediterráneos "Al-Masāq. Studia Arabo-Islamica Mediterranea", fundada por el Prof. Dionisius A. Agius, de la Universidad de Leeds (Department of Modern Arabic Studies, University of Leeds, Leeds LS2 9JT, Gran Bretaña). El Dr. Epalza participó con un artículo en el volumen I, 1988.

BOLETÍN "ARABISMO ALICANTINO». Desde diciembre 1987 y con una periodicidad trimestral, la División de Estudios Árabes e Islámicos de la Universidad de Alicante publica un boletín informativo bilingüe, castellano y catalán, sobre cuantas actividades relacionadas con la cultura árabe se realizan en la provincia de Alicante o relacionadas con ella. Está redactado por los Sres. Epalza, Franco y Srta. Martín. Distribuido gratuitamente entre estudiantes y simpatizantes, ha tirado en 1988 cuatro números, con una tirada de 700 ejemplares cada uno.

CONFERENCIAS DE LA DIVISIÓN DE ESTUDIOS ÁRABES E ISLÁMICOS. Dentro de los cursos para postgraduados de "máster" "Estudios Euroárabes" (ver SHARQ AL-ANDALUS. ESTUDIOS ÁRABES número 4, p. 374) impartieron conferencias:

General José Timón Lara: "Experiencias de la descolonización militar española en el Norte de África» (05-03-1988). 
Dr. José Valdivia Valor: "Estudios españoles sobre mística musulmana» (16-04-1988).

Prof. Ahmad Kamel Berraghda: "Los estudios hispánicos en Argelia» y "La Independencia argelina en la prensa y las literaturas hispánicas" (30-04-1988).

Dr. José Pérez Lázaro: "Las relaciones culturales hispanoárabes en la actualidad" (11-06-1988), con la que se clausuró este primer año del máster.

El 4 de mayo presentaron el libro "A vuelo de pájaro sobre Marruecos", su autora Concha López Sarasúa, el escritor Mohamed Chakor de la Agencia Maroc Presse en Madrid y el Dr. Epalza.

Durante la segunda semana de marzo, el Dr. Epalza presentó en diversos cursos del Departamento la película de Carles Mira "Daniya», sobre historia catalano-árabe del siglo Xl, de la que ha sido asesor.

CONFERENCIAS DEL 750 ANIVERSARIO DE LA CONQUISTA DE VALENCIA. Dentro del ciclo de conferencias organizado por el Consell Valencià de Cultura y por la Facultad de Filosofía y Letras, durante el último trimestre de 1988, se dieron en la Facultad las siguientes conferencias de tema árabe, cuyos textos se recogerán en un volumen:

Dr. Federico Corriente: "Las jarchas valencianas" (18-10-1988).

Dra. María Jesús Rubiera: «Aspectes de la poesia àrab valenciana: Paradís i Paradis Perdut” (19-10-1988).

Dr. Míkel de Epalza: «L'esplendor de les Taifes Valencianes» (28-11-1988).

Dr. Juan Vernet: "La ciència abans de la Conquesta" (29-11-1988).

Dr. Robert I. Burns (no pudo dar la conferencia, por coincidir con la huelga general del 14 de diciembre).

Dr. Thomas Glick: "La técnica de riegos antes y después de la Conquista» (15-12-1988). 